\title{
On the $c_{0}$-equivalence and permutations of series
}

\section{Artur Bartoszewicz ${ }^{1} \cdot$ Włodzimierz Fechner $^{1}$ (D ) Aleksandra Świątczak ${ }^{1}$. Agnieszka Widz ${ }^{1}$}

Received: 23 August 2020 / Accepted: 22 December 2020 / Published online: 26 January 2021

(C) The Author(s) 2021

\section{Abstract}

Assume that a convergent series of real numbers $\sum_{n=1}^{\infty} a_{n}$ has the property that there exists a set $A \subseteq \mathbb{N}$ such that the series $\sum_{n \in A} a_{n}$ is conditionally convergent. We prove that for a given arbitrary sequence $\left(b_{n}\right)$ of real numbers there exists a permutation $\sigma: \mathbb{N} \rightarrow \mathbb{N}$ such that $\sigma(n)=n$ for every $n \notin A$ and $\left(b_{\infty}\right)$ is $c_{0}$-equivalent to a subsequence of the sequence of partial sums of the series $\sum_{n=1}^{\infty} a_{\sigma(n)}$. Moreover, we discuss a connection between our main result with the classical Riemann series theorem.

Keywords $c_{0}$-Equivalence $\cdot$ Center of distances $\cdot$ Potentially convergent series $\cdot$ von Neumann's theorem $\cdot$ Riemann rearrangement theorem $\cdot$ Hypernumber

Mathematics Subject Classification 40A05 - 40A35

\section{Communicated by Vladimir Rakocevic.}

Włodzimierz Fechner

wlodzimierz.fechner@p.lodz.pl

Artur Bartoszewicz

artur.bartoszewicz@p.lodz.pl

Aleksandra Świątczak

aleswi97@gmail.com

Agnieszka Widz

AgnieszkaWidzENFP@gmail.com

1 Institute of Mathematics, Lodz University of Technology, ul. Wólczańska 215, 90-924 Łódź,

Poland 


\section{Introduction}

By $\mathbb{R}$ we denote the set of reals and $\mathbb{N}=\{1,2, \ldots\}$. By an ideal, or set-ideal to distinguish from an algebraic ideal, we mean a family $\mathcal{I} \subset \mathcal{P}(\mathbb{N})$ which is closed under finite unions and contains all subsets of each of its members. An ideal is proper if it does not contain $\mathbb{N}$ and a proper ideal is admissible if it contains all singletons $\{n\}$. An ideal $\mathcal{I}$ is called summable if there exists a sequence of positive numbers $\left(a_{n}\right)$ such that $\sum_{n=1}^{\infty} a_{n}=+\infty$ and $A \in \mathcal{I}$ if and only if $\sum_{n \in A} a_{n}<+\infty$.

Next, for a given ideal $\mathcal{I}$ we say that a sequence $\left(x_{n}\right)$ of real numbers is $\mathcal{I}$ -bounded if there exists a constant $\lambda>0$ such that the set $\left\{n:\left|x_{n}\right|>\lambda\right\}$ belongs to $\mathcal{I}$. The set of all $\mathcal{I}$-bounded sequences is denoted by $\ell^{\infty}(\mathcal{I})$ and is equipped with a semi-norm given by

$$
\left\|\left(x_{n}\right)\right\|_{\infty}^{\mathcal{I}}=\inf \left\{\lambda>0:\left\{n \in \mathbb{N}:\left|x_{n}\right|>\lambda\right\} \in \mathcal{I}\right\} .
$$

Further, a sequence $\left(x_{n}\right)$ is termed $\mathcal{I}$-convergent to some $x_{0} \in \mathbb{R}$ if for every $\varepsilon>0$ the set $\left\{n \in \mathbb{N}:\left|x_{n}-x_{0}\right|>\varepsilon\right\}$ belongs to $\mathcal{I}$. The set of all $\mathcal{I}$-convergent sequences is denoted by $c(\mathcal{I})$ and by $c_{0}(\mathcal{I})$ we mean its subspace of all sequences which are $\mathcal{I}$ -convergent to 0 . We say that two sequences $\left(x_{n}\right)$ and $\left(y_{n}\right)$ of elements of a metric space $(X, d)$ are $c_{0}$-equivalent if

$$
\lim _{n \rightarrow \infty} d\left(x_{n}, y_{n}\right)=0 .
$$

This relation is most often considered in the case of $\ell^{\infty}$ or of a compact space $X$. Below we illustrate both situations by recalling some applications of this notion.

Theorem 1.1 (Semadeni [17, Theorem 4.2.2, p.77]) The space $\ell^{\infty} / c_{0}$ is isometrically isomorphic to the space of continuous functions on the remainder $\beta \mathbb{N} \backslash \mathbb{N}$ of the Čech-Stone compactification of the discrete space $\mathbb{N}$.

In Ref. [4], the authors have generalized this by proving a set-ideal version of the above theorem. Given an admissible ideal $\mathcal{I} \subset \mathcal{P}(\mathbb{N})$ by $P_{\mathcal{I}}$ we denote the set of all proper ultrafilters $p$ in $\mathcal{P}(\mathbb{N})$ such that $\bigcap p=\varnothing$ and the family $p^{*}$ of the complements of all elements of $p$ contains $\mathcal{I}$. The family $P_{\mathcal{I}}$ is a closed subset of $\beta \mathbb{N}$ (see Balcar and Simon [1]) and, therefore, it makes sense to consider the space $C\left(P_{\mathcal{I}}\right)$ of all continuous functions on $P_{\mathcal{I}}$.

Theorem 1.2 (Bartoszewicz et al. [4, Theorem 1]) For every admissible ideal $\mathcal{I} \subset \mathcal{P}(\mathbb{N})$ the spaces $\ell^{\infty}(\mathcal{I}) / c_{0}(\mathcal{I})$ and $C\left(P_{\mathcal{I}}\right)$ are isometrically isomorphic.

Roughly speaking, the above statement says that in Semadeni's result one can use the seminorm $\|\cdot\|_{\infty}^{\text {Fin }}$ on the space $\ell^{\infty}$, where Fin is the ideal of finite subsets of $\mathbb{N}$, obtaining the same effect.

According to a well-known theorem of von Neumann, two sequences $\left(x_{n}\right),\left(y_{n}\right)$ of elements of a compact metric space have the same set of limit points if and 
only if $\left(x_{n}\right)$ is $c_{0}$-equivalent to some permutation of $\left(y_{n}\right)$. Recently Bielas et al. [6, Theorem 2.1] have generalized one implication of the von Neumann's theorem using the so-called "back-and-forth" method. In a paper by Banakiewicz et al. [3], the reverse implication to this theorem was proved. Given a metric space $(X, d)$ the center of distances of $X$ is defined as

$$
Z(X)=\{\alpha \geq 0: \forall x \in X, \exists y \in X \text { such that } d(x, y)=\alpha\} .
$$

Theorem 1.3 ([3, Theorem 1.2]) Assume that $(X, d)$ is a compact metric space, $\left(x_{n}\right),\left(y_{n}\right)$ are sequences of elements of $X$ which have the same set of limit points $A$. Then for a given $\alpha \geq 0$ there exists a permutation $\sigma: \mathbb{N} \rightarrow \mathbb{N}$ such that

$$
\lim _{n \rightarrow \infty} d\left(x_{n}, y_{\sigma(n)}\right)=\alpha
$$

if and only if $\alpha \in Z(A)$.

From the above statement, one can easily derive the less trivial implication of the von Neumann's theorem, which corresponds to the case $\alpha=0$. Moreover, we can formulate an immediate corollary.

Corollary 1.1 Assume that $(X, d)$ is a compact metric space and $\left(x_{n}\right)$ is a sequence of elements of $X$ with the set of limit points A. Then there exists a permutation $\sigma: \mathbb{N} \rightarrow \mathbb{N}$ such that

$$
\lim _{n \rightarrow \infty} d\left(x_{n}, x_{\sigma(n)}\right)=\alpha
$$

if and only if $\alpha \in Z(A)$.

The aim of this note is to show an application of the $c_{0}$-equivalence in the case of unbounded sequences. Our main theorem can be treated as an extension of the Riemann rearrangement theorem.

\section{Main result}

For an arbitrary number $a \in \mathbb{R}$, we denote $a^{+}=\max \{a, 0\}$ and $a^{-}=a^{+}-a$. A series $\sum_{n=1}^{\infty} a_{n}$ is called potentially conditionally convergent if the following three conditions hold:
(a) $\sum_{n_{\infty} 1}^{\infty} a_{n}^{+}=+\infty$,
(b) $\sum_{n=1} a_{n}^{-}=+\infty$,
(c) $\lim _{n \rightarrow \infty} a_{n}=0$. 
It is clear that every conditionally convergent series is potentially conditionally convergent.

Our main theorem reads.

Theorem 2.1 Let $\left(a_{n}\right)$ be a sequence of real numbers such that the series $\sum^{\infty} a_{n}$ is convergent and assume that there exists a set $A \subseteq \mathbb{N}$ such that the series $\sum_{n \in A}^{n=1} a_{n}$ is conditionally convergent. Next, let $\left(b_{n}\right)$ be an arbitrary sequence of real numbers. Then there exist a permutation $\sigma: \mathbb{N} \rightarrow \mathbb{N}$ satisfying $\sigma(n)=n$ for every $n \notin A$ and $a$ subsequence of the sequence of partial sums of the series $\sum_{n=1}^{\infty} a_{\sigma(n)}$ which is $c_{0}$-equivalent to the sequence $\left(b_{n}\right)$.

Proof Note that the series $\sum_{n=1}^{\infty} a_{n}$ is conditionally convergent. In particular, we have $\sum_{n=1}^{\infty} a_{n}^{+}=+\infty$ and $\sum_{n=1}^{\infty} a_{n}^{-}=+\infty$.

We will inductively construct a bijection $\sigma: \mathbb{N} \rightarrow \mathbb{N}$ and a strictly increasing sequence of positive integers $\left(k_{n}\right)$ such that $S_{k_{n}}-b_{n}$ tends to 0 as $n \rightarrow \infty$, where $S_{k_{n}}=\sum_{j=1}^{k_{n}} a_{\sigma(j)}$

First, we put $\sigma(j)=j$ for all $j \notin A$. Let us put $k_{0}=0$ and observe that for $n=0$ the inductive assumptions are trivially satisfied, so we have the base for our induction.

If $n>0$ let us assume that we have found positive integers $k_{1}, k_{2}, \ldots, k_{n}$ which form a strictly increasing sequence, the values $\sigma(j)$ are defined for every integer $j \in\left\{1, \ldots, k_{n}\right\}$, one has $\sigma(j) \in A$ whenever $j \in A$ and

$$
\left|S_{k_{m}}-b_{m}\right| \leq \frac{1}{m}, \text { for } \quad m \leq n \text {. }
$$

If $n=0$, then we do not assume that (2.1) holds and, moreover, any value of $\sigma$ is not defined at the moment. All subsequent arguments remain the same.

In case $n>0$ for $m<n$, let $k_{m}^{*}$ be the smallest integer which is greater than $k_{m}$ and belongs to $A$. Let us assume, moreover, that $\sigma\left(k_{m}^{*}\right) \in A$ is the smallest positive integer which does not belong to the set $\left\{\sigma(j): j \leq k_{m}\right\}$. This assumption will be used to obtain the surjectivity of $\sigma$.

Since the series $\sum_{j \notin A} a_{j}$ is convergent, then it satisfies the Cauchy condition. Therefore, there exists a positive integer $k_{n+1}^{\prime}>k_{n}$ such that for $q>k_{n+1}^{\prime}$ one has

$$
\left|\sum_{j=1, j \notin A}^{q} a_{j}-\sum_{j=1, j \notin A}^{k_{n+1}^{\prime}} a_{j}\right|=\left|\sum_{j=k_{n+1}^{\prime}+1, j \notin A}^{q} a_{j}\right| \leq \frac{1}{2(n+1)} .
$$

Now, we are able to define a positive integer $k_{n+1}>k_{n+1}^{\prime}$ and values $\sigma(j)$ for $k_{n}+1 \leq j \leq k_{n+1}$ in such a way that the following two conditions hold: 
(A) $\sigma\left(k_{n}^{*}\right)$ is the smallest positive integer in $A$ which does not appear in the set $\left\{\sigma(j): j \leq k_{n}, j \in A\right\}$

(B) the following estimate holds true:

$$
\left|\sum_{j=k_{n}+1, j \in A}^{k_{n+1}} a_{\sigma(j)}-\left(b_{n+1}-\sum_{j=1}^{k_{n}} a_{\sigma(j)}-\sum_{j=k_{n}+1, j \notin A}^{k_{n+1}^{\prime}} a_{j}\right)\right| \leq \frac{1}{2(n+1)} .
$$

Indeed, the series $\sum_{j \in A} a_{j}$ is conditionally convergent. Hence, any real number $r$ can be estimated by the partial sums of a series $\sum_{j \in A} a_{\sigma_{1}(j)}$ for some permutation $\sigma_{1}: \mathbb{N} \rightarrow \mathbb{N}$. Obviously, we can take $\sigma_{1}(j)=\sigma(j)$ for $j \leq k_{n}$. So for some $k_{n+1}$, we can have

$$
\left|\sum_{j=1, j \in A}^{k_{n+1}} a_{\sigma_{1}(j)}-\left(b_{n+1}-\sum_{j=1, j \notin A}^{k_{n+1}^{\prime}} a_{j}\right)\right| \leq \frac{1}{2(n+1)},
$$

which, defining $\sigma(j):=\sigma_{1}(j)$ for $k_{n}+1 \leq j \leq k_{n+1}$, gives us (2.3). Of course we can assume that $k_{n+1}$ is greater than $k_{n+1}^{\prime}$. Making use of (2.2) and (2.3), we obtain

$$
\begin{aligned}
\left|S_{k_{n+1}}-b_{n+1}\right|= & \left|\sum_{j=1}^{k_{n+1}} a_{\sigma(j)}-b_{n+1}\right| \\
= & \left|\sum_{j=1}^{k_{n}} a_{\sigma(j)}+\sum_{j=k_{n}+1, j \in A}^{k_{n+1}} a_{\sigma(j)}+\sum_{j=k_{n}+1, j \notin A}^{k_{n+1}} a_{j}-b_{n+1}\right| \\
= & \mid \sum_{j=1}^{k_{n}} a_{\sigma(j)}+\sum_{j=k_{n}+1, j \in A}^{k_{n+1}} a_{\sigma(j)}+\sum_{j=k_{n}+1, j \notin A}^{k_{n+1}} a_{j} \\
& -\sum_{j=k_{n}+1, j \notin A}^{k_{n+1}^{\prime}} a_{j}+\sum_{j=k_{n}+1, j \notin A}^{k_{n+1}^{\prime}} a_{j}-b_{n+1} \mid \\
& +\left|\sum_{j=k_{n}+1, j \in A}^{k_{n+1}} a_{\sigma(j)}-\right| b_{n+1}-\sum_{j=1}^{k_{n}} a_{\sigma(j)}-\sum_{j=k_{n}+1, j \notin A}^{k_{n+1}^{\prime}} a_{j} \mid \\
& +\left|\sum_{j=k_{n+1}^{\prime}+1, j \notin A}^{k_{n+1}} a_{j}\right| \leq \frac{1}{2(n+1)}+\frac{1}{2(n+1)}=\frac{1}{(n+1)} .
\end{aligned}
$$

Finally, let us observe that our definition of the value $\sigma\left(k_{n}^{*}\right)$ guarantees the surjectivity of $\sigma$.

Let us formulate some corollaries. 
Corollary 2.1 Let $\left(a_{n}\right)$ be a sequence of real numbers such that the series $\sum_{n=1}^{\infty} a_{n}$ is potentially conditionally convergent. Next, let $\left(b_{n}\right)$ be an arbitrary sequence of real numbers. Then there exists a permutation $\sigma: \mathbb{N} \rightarrow \mathbb{N}$ such that the sequence $\left(b_{n}\right)$ is $c_{0}$-equivalent to some subsequence of the sequence of partial sums of the series $\sum_{n=1} a_{\sigma(n)}$

Proof There exists a permutation $\pi: \mathbb{N} \rightarrow \mathbb{N}$ such that the series $\sum_{n=1}^{\infty} a_{\pi}(n)$ is convergent. Apply our theorem for this series and the set $A=\mathbb{N}$ to get a permutation $\sigma^{\prime}: \mathbb{N} \rightarrow \mathbb{N}$ such that the sequence $\left(b_{n}\right)$ is $c_{0_{\infty}^{-}}$-equivalent to some subsequence of the sequence of partial sums of the series $\sum_{n=1} a_{\left(\sigma^{\prime} \circ \pi\right)(n)}$. To finish the proof take $\sigma=\sigma^{\prime} \circ \pi$

Wilczyński [18] has proved that for every conditionally convergent series $\sum_{n=1}^{\infty} a_{n}$, there exists a set $A$ of density zero such that the series $\sum_{n=1, n \in A}^{\infty} a_{n}$ is conditionally convergent. He posed an open problem in Ref. [18] to characterize all set ideals $\mathcal{I}$ with the property that for every conditionally convergent series $\sum_{n=1}^{\infty} a_{n}$, there exists a set $A \in \mathcal{I}$ such that the series $\sum_{n=1, n \in A}^{\infty} a_{n}$ is conditionally convergent. Filipów and Szuca [11] answered this problem and they proved that a set ideal $\mathcal{I}$ has the above-mentioned property if and only if it cannot be extended to a summable ideal.

On joining [18, Lemma] with our main result, we can take the set $A$ to be of density zero.

Corollary 2.2 Let $\left(a_{n}\right)$ be a sequence of real numbers such that the series $\sum_{n=1}^{\infty} a_{n}$ is conditionally convergent. Next, let $\left(b_{n}\right)$ be an arbitrary sequence of real numbers. Then there exists a permutation $\sigma: \mathbb{N} \rightarrow \mathbb{N}$ such that

$$
\lim _{n \rightarrow \infty} \frac{1}{n}|\{n \in \mathbb{N}: \sigma(n) \neq n\}|=0
$$

and the sequence $\left(b_{n}\right)$ is $c_{0}$-equivalent to some subsequence of the sequence of partial sums of the series $\sum_{n=1}^{\infty} a_{\sigma(n)}$.

It is worth to note a connection of our results with the Riemann rearrangement theorem. One can reformulate this classical result as follows:

Theorem 2.2 (Riemann rearrangement theorem) Let $\left(a_{n}\right)$ be a sequence of real numbers such that the series $\sum_{n=1}^{\infty} a_{n}$ is conditionally convergent, let $b \in \mathbb{R} \cup\{ \pm \infty\}$ be arbitrary and let $\left(b_{n}\right)$ be a sequence of real numbers tending to $b$. Then there exists a 
permutation $\sigma: \mathbb{N} \rightarrow \mathbb{N}$ such that the sequence $\left(b_{n}\right)$ is $c_{0}$-equivalent to the sequence of partial sums of the series $\sum_{n=1}^{\infty} a_{\sigma(n)}$.

It is easy to note two differences between our Corollary 2.1 and the Riemann rearrangement theorem. First, we do not assume that the sequence $\left(b_{n}\right)$ is convergent (in fact, it can be even unbounded in our settings). But in return for it, in general only a subsequence of the sequence of partial sums of the series $\sum_{n=1}^{\infty} a_{\sigma(n)}$ is $c_{0}$-equivalent to $\left(b_{n}\right)$.

Recently, several results related to the Riemann rearrangement theorem and conditional convergence of series were published by several authors, see $[2,5,9$, 10, 12-16, 19-21].

We will terminate the paper with a generalization of an interesting result of Burgin [7, Corollary 4.6] which extends the classical Riemann rearrangement theorem to the case of the so-called hypernumbers. However, the original proof of Bounded Riemann Series Theorem (BRST, [7, Theorem 4.5]) contains a gap which cannot be removed directly. We give some counterexamples to this statement. We provide an alternative argument and at the same time we obtain a slightly more general statement than the Burgin's Generalized Riemann Series Theorem (GRST, [7, Corollary 4.6]).

Hypernumbers have been founded by Burgin and they provide an extension of the real line. This extension is of importance in many areas of mathematics and in physics. The elegant and deep theory of hypernumbers allows one in particular to differentiate in an extended sense every real function. We recall here only basic notions we need in what follows. For a comprehensive study of the topic, the reader is referred to the excellent monograph by Burgin [8]. Let $\mathbb{R}^{\mathbb{N}}$ stand for the set of all sequences of real numbers. Following Burgin's notation, the set of $c_{0}$ -equivalence classes of $\mathbb{R}^{\mathbb{N}}$ is denoted by $\mathbb{R}_{\omega}$ and its elements are called hypernumbers. Given a sequence $\left(a_{n}\right) \in \mathbb{R}^{\mathbb{N}}$, by $\operatorname{Hn}\left(\left(a_{n}\right)\right)$, we denote its equivalence class, i.e. the hypernumber which is represented by this sequence. In particular, every real number can be identified with a constant sequence equal to it and, therefore, the real line $\mathbb{R}$ is a proper subset of the set of all hypernumbers $\mathbb{R}_{\omega}$. A hypernumber is called bounded if it is generated by a bounded sequence. If $\alpha, \beta \in \mathbb{R}_{\omega}$, then we say that $\beta$ is a subnumber of $\alpha$, which is denoted by $\beta \Subset \alpha$, if there exist two sequences $\left(a_{n}\right),\left(b_{n}\right)$ such that $\left(b_{n}\right)$ is a subsequence of $\left(a_{n}\right), \alpha=\operatorname{Hn}\left(\left(a_{n}\right)\right)$ and $\beta=\operatorname{Hn}\left(\left(b_{n}\right)\right)$.

Following Burgin [7], given a series of real numbers $\sum_{n=1}^{\infty} a_{n}$, convergent or not, we say that a series $\sum_{n=1}^{\infty} b_{n}$ is a quotient series of $\sum_{n=1}^{\infty} a_{n}$ if there is a surjective map $p: \mathbb{N} \rightarrow \mathbb{N}$ such that for every $i \in \mathbb{N}$ the set $p^{-1}(i)$ consists of a finite number of consecutive elements of $\mathbb{N}$ and $b_{i}=\sum_{j \in p^{-1}(i)} a_{j}$. If additionally the map $p$ is monotone, then we speak about a monotone quotient series.

Burgin [7] introduced the concept of analytical sums of a series as a hypernumber generated by its partial sums. For an arbitrary series of real numbers $\sum_{n=1}^{\infty} a_{n}$, we 
write $\operatorname{an}\left(\sum_{n=1}^{\infty} a_{n}\right)=\operatorname{Hn}\left(\left(A_{n}\right)\right)$, where $A_{n}=\sum_{i=1}^{n} a_{i}$ for $n \in \mathbb{N}$. If the series $\sum_{n=1}^{\infty} a_{n}$ is convergent, then its analytical sum coincides with its topological sum, i.e. the real number equal to its sum, which is denoted by $\operatorname{top}\left(\sum_{n=1}^{\infty} a_{n}\right)$. For series divergent to $\pm \infty$ one can also speak about its topological sums.

One can observe that if $\alpha, \beta \in \mathbb{R}_{\omega}$ satisfy

$$
\alpha=\operatorname{an}\left(\sum_{n=1}^{\infty} a_{n}\right), \quad \beta=\operatorname{an}\left(\sum_{n=1}^{\infty} b_{n}\right)
$$

for some $\left(a_{n}\right),\left(b_{n}\right) \in \mathbb{R}^{\mathbb{N}}$, then the series $\sum_{n=1}^{\infty} b_{n}$ is a monotone quotient series of $\sum_{n=1}^{\infty} a_{n}$ if and only if $\beta \Subset \alpha$ (see [7, Lemma 4.4]).

The Bounded Riemann Series Theorem (BRST) [7, Theorem 4.5] deals with a series $\sum_{n=1}^{\infty} a_{n}$ which is bounded and not absolutely convergent. Thus, $\operatorname{an}\left(\sum_{n=1}^{\infty} a_{n}\right)$ is a bounded hypernumber. BRST says that for every hypernumber $\alpha \underset{\infty}{\in} \mathbb{R}_{\omega}$ there exists a monotone quotient series $\sum_{n=1}^{\infty} d_{n}$ of $\sum_{n=1}^{\infty} a_{n}$, a permutation $\sum_{n=1}^{\infty} b_{n}$ of $\sum_{n=1}^{\infty} d_{n}$ and a monotone quotient series $\sum_{n=1}^{\infty} c_{n}$ of $\sum_{n=1}^{\infty} b_{n}$ such that $\alpha=\operatorname{an}\left(\sum_{n=1}^{\infty} c_{n}\right)$. This statement is in general not true, which is exhibited by examples below. Moreover, an inspection of the proof of [7, Theorem 4.5] allows us to detect an invalid argument that was used. Namely, it is not true that a monotone quotient series of a series which is not absolutely convergent is necessarily not absolutely convergent. Consequently, Riemann rearrangement theorem cannot be applied. Our examples shed some more light on the situation.

Example 2.1 Let $a_{n}=(-1)^{n+1}$ for $n \in \mathbb{N}$. Then the series $\sum_{n=1}^{\infty} a_{n}$ is not absolutely convergent (in fact it is divergent) and its analytical sum is a bounded hypernumber. Thus, the assumptions of BRST are satisfied. Note that every converging subsequence of its partial sums $A_{n}$ must be from some point equal either to 0 or 1 . Therefore, every monotone quotient series $\sum_{n=1}^{\infty} d_{n}$ of $\sum_{n=1}^{\infty} a_{n}$ which is convergent converges to 0 or 1 . Thus, every permutation $\sum_{n=1}^{\infty} b_{n}$ of $\sum_{n=1}^{\infty} d_{n}$ is either equal to the zero series, or to a series which contains 1 at one place. Consequently, every monotone quotient series $\sum_{n=1}^{\infty} c_{n}$ of $\sum_{n=1}^{\infty} b_{n}$ is also either equal to the zero series, or it contains 1 at one place. Two in a sense typical examples of monotone quotient series of $\sum_{n=1}^{\infty} a_{n}$ are $0+0+\ldots$ and $1+0+0+\ldots$. The first case can be obtained by the projection $j_{0}: \mathbb{N} \rightarrow \mathbb{N}$ given by $j_{0}(2 k-1)=j_{0}(2 k)=k$ for $k \in \mathbb{N}$, whereas the second one by the projection $j_{1}: \mathbb{N} \rightarrow \mathbb{N}$ given by $j_{1}(1)=1$ and $j_{1}(2 k)=j_{1}(2 k+1)=k$ for $k \in \mathbb{N}$. Thus, only two hypernumbers $\alpha$, namely 0 and 1 can be achieved from the series $\sum_{n=1}^{\infty} a_{n}$ by means of the proof of Burgin's BRST. Note, however, that every hyper- 
number which is of the form $\operatorname{Hn}\left(q_{n}\right)$ with all $q_{n}$ being integers can be obtained as a monotone quotient series of a permutation of $\sum_{n=1}^{\infty} a_{n}$. Moreover, any hypernumber which is not of this form cannot be obtained as a monotone quotient series of $\sum_{n=1}^{\infty} a_{n}$. Example 2.2 Let $a_{n}=(-1)^{n+1} / n$ for $n \in \mathbb{N}$. Then the series $\sum_{n=1}^{\infty} a_{n}$ is conditionally convergent (to $\ln 2$ ). Again, the assumptions of BRST are satisfied. This time there exists a monotone quotient series $\sum_{n=1}^{\infty} d_{n}$ of $\sum_{n=1}^{\infty} a_{n}$ which is absolutely convergent. Indeed, one can take any projection from the previous example, if we take $j_{0}: \mathbb{N} \rightarrow \mathbb{N}$ given by $j_{0}(2 k-1)=j_{0}(2 k)=k$ for $k \in \mathbb{N}$, then we obtain a convergent series of positive numbers. But in contrast to the previous example, this time it is possible to achieve every hypernumber as a monotone quotient series of a permutation of the original one. This is a straightforward consequence of Corollary 2.3.

Corollary 2.3 Assume that $\alpha \in \mathbb{R}_{\omega}$ is an arbitrary hypernumber and $\left(a_{n}\right)$ is a sequence of real numbers such that the series $\sum_{n=1}^{\infty} a_{n}$ is potentially conditionally convergent. Then there exists a permutation $\sigma: \mathbb{N} \rightarrow \mathbb{N}$ such that $\alpha$ is equal to a monotone quotient series of the series $\sum_{n=1}^{\infty} a_{\sigma(n)}$.

Proof Follows immediately from our Theorem 2.1.

Open Access This article is licensed under a Creative Commons Attribution 4.0 International License, which permits use, sharing, adaptation, distribution and reproduction in any medium or format, as long as you give appropriate credit to the original author(s) and the source, provide a link to the Creative Commons licence, and indicate if changes were made. The images or other third party material in this article are included in the article's Creative Commons licence, unless indicated otherwise in a credit line to the material. If material is not included in the article's Creative Commons licence and your intended use is not permitted by statutory regulation or exceeds the permitted use, you will need to obtain permission directly from the copyright holder. To view a copy of this licence, visit http://creativecommons.org/licen ses/by/4.0/.

\section{References}

1. Balcar, B., Simon, P.: Appendix on general topology. In: Handbook of Boolean Algebras, vol. 3, pp. 1239-1267. North-Holland, Amsterdam (1989)

2. Banakiewicz, M., Hanson, B., Pierce, P., Prus-Wiśniowski, F.: A Riemann-type theorem for segmentally alternating series. Bull. Iran. Math. Soc. 44(5), 1303-1314 (2018)

3. Banakiewicz, M., Bartoszewicz, A., Prus-Wiśniowski, F.: The center of distances of some multigeometric series. Preprint at arXiv: 1907.03800v1 [math.CA] (2019)

4. Bartoszewicz, A., Głąb, S., Wachowicz, A.: Remarks on ideal boundedness, convergence and variation of sequences. J. Math. Anal. Appl. 375(2), 431-435 (2011) 
5. Bartoszewicz, A., Głąb, S., Marchwicki, J.: Achievement sets of conditionally convergent series. Colloq. Math. 152(2), 235-254 (2018)

6. Bielas, W., Plewik, S., Walczyńska, M.: On the center of distances. Eur. J. Math. 4(2), 687-698 (2018)

7. Burgin, M.: Inequalities in series and summation in hypernumbers. In: Dragomir, S.S., Sofo, A. (eds.) Advances in Inequalities for Series, pp. 89-120. Nova Science Publishers, Hauppauge, New York (2008)

8. Burgin, M.: Hypernumbers and extrafunctions. Extending the classical calculus, series $=$ SpringerBriefs in Mathematics, pp viii+160. Springer, New York (2012)

9. Charatonik, W.J., Samulewicz, A., Wituła, R.: Limit sets in normed linear spaces. Colloq. Math. 147(1), 35-42 (2017)

10. Dybskiy, Y., Slutsky, K.: Riemann rearrangement theorem for some types of convergence. J. Math. Anal. Appl. 373(2), 605-613 (2011)

11. Filipów, R., Szuca, P.: Rearrangement of conditionally convergent series on a small set. J. Math. Anal. Appl. 362(1), 64-71 (2010)

12. Freniche, F.J.: On Riemann's rearrangement theorem for the alternating harmonic series. Am. Math. Mon. 117(5), 442-448 (2010)

13. Grahl, J., Nevo, S.: On Riemann's theorem about conditionally convergent series. N. Z. J. Math. 43, 85-93 (2013)

14. Jóźwik, I., Terepeta, M.: Polish glance on the Riemann Rearrangement Theorem. Wiadomości Matematyczne 55(1), 143-157 (2019) (in Polish)

15. Marchwicki, J.: Achievement sets and sum ranges with ideal supports. Filomat 32(14), 4911-4922 (2018)

16. Prus-Wiśniowski, F.: Two refinements of the Riemann derangement theorem, conference $=$ Real functions, density topology and related topics, pp. 165-172. Łódź Univ. Press, Łódź (2011)

17. Semadeni, Z.: Banach spaces of continuous functions, vol. I, 584 pp. Monografie Matematyczne, Tom 55, PWN_-Polish Scientific Publishers, Warsaw (errata insert) (1971)

18. Wilczyński, W.: On Riemann derangement theorem. Słup. Pr. Mat.-Fiz. 4, 79-82 (2007)

19. Wituła, R., Hetmaniok, E., Kaczmarek, K.: On series whose rearrangements possess discrete sets of limit points. J. Appl. Anal. 20(1), 93-96 (2014)

20. Wituła, R.: Permutations preserving the convergence or the sum of series-a survey, conference= Monograph on the occasion of 100th birthday anniversary of Zygmunt Zahorski, Wydaw, pp. 169190. Politech. Śl., Gliwice (2015)

21. Wituła, R., Hetmaniok, E., Słota, D.: Some new facts about group mathcalG generated by the family of convergent permutations. Open Math. 15(1), 568-577 (2017) 